PULMONARY HYPERTENSION

\title{
Survival in patients with class III idiopathic pulmonary arterial hypertension treated with first line oral bosentan compared with an historical cohort of patients started on intravenous epoprostenol
}

\author{
O Sitbon, V V McLaughlin, D B Badesch, R J Barst, C Black, N Galiè, M Humbert, \\ M Rainisio, L J Rubin, G Simonneau
}

See end of article for authors' affiliations

Correspondence to: Dr O Sitbon, Centre des Maladies Vasculaires Pulmonaires, Hôpital Antoine Béclère, Université Paris-Sud, 157 rue de la Porte de Trivaux, 92141 Clamart, France; olivier. sitbon@abc.aphp.fr

Received 19 January 2005 Accepted 3 July 2005 Published Online First 29 July 2005

Background: The oral dual endothelin receptor antagonist bosentan improves exercise capacity and delays clinical worsening in patients with pulmonary arterial hypertension, but its use could delay starting intravenous epoprostenol, a life saving treatment.

Methods: Survival in patients with functional class III idiopathic pulmonary arterial hypertension (PAH) treated with bosentan in clinical trials was compared with historical data from similar patients treated with epoprostenol in the clinic. Statistical methods were used to adjust for possible underlying differences between the two groups.

Results: Baseline factors for the 139 patients treated with bosentan and the 346 treated with epoprostenol suggested that the epoprostenol cohort had more severe disease-that is, a lower cardiac index $(2.01 \mathrm{v}$ $2.39 \mathrm{l} / \mathrm{min} / \mathrm{m}^{2}$ ) and higher pressures and resistance. Kaplan-Meier survival estimates after 1 and 2 years were $97 \%$ and $91 \%$, respectively, in the bosentan cohort and $91 \%$ and $84 \%$ in the epoprostenol cohort. Cox regression analyses adjusting for differences in baseline factors showed a greater probability of death in the epoprostenol cohort (hazard ratio 2.2 (95\% confidence interval 1.2 to 4.0 ) in the model adjusted for haemodynamics). Alternative regression analyses and analyses to adjust for different data collection dates gave consistently similar results. When matched cohorts of 83 patients each were selected, survival estimates were similar. In the bosentan cohort $87 \%$ and $75 \%$ of patients followed for 1 and 2 years, respectively, remained on monotherapy.

Conclusions: No evidence was found to suggest that initial treatment with oral bosentan, followed by or with the addition of other treatment if needed, adversely affected the long term outcome compared with initial intravenous epoprostenol in patients with class III idiopathic PAH.

$\mathrm{S}$ everal treatments of pulmonary arterial hypertension (PAH) are now approved in North America (intravenous epoprostenol, subcutaneous treprostinil, oral bosentan) and Europe (intravenous epoprostenol, intravenous or inhaled iloprost, oral bosentan). With the exception of recent data from patients receiving prolonged treatment with epoprostenol, $^{12}$ the long term effects of these treatments are unknown. There is a need for long term observational studies to evaluate the different treatments in terms of survival, side effects, quality of life, and costs. Ideally, the best way to compare treatments is by head to head comparisons but, in the absence of such data, the choice of optimal treatment is currently dictated by clinical experience and drug availability, as well as patient preference.

Two 12 week open label, randomised studies have shown that a continuous intravenous infusion of epoprostenol $\left(\right.$ Flolan $^{\circledR}$ ) improves exercise capacity, quality of life, and haemodynamics in patients with functional class III-IV idiopathic $\mathrm{PAH}^{3}$ and PAH related to scleroderma. ${ }^{4}$ In addition, analyses of large cohorts of patients with idiopathic PAH have shown that long term survival is improved in epoprostenol treated patients compared with either predicted survival ${ }^{1}$ or an historical control group. ${ }^{2}$ These findings have led to the suggestion of initiating treatment with continuous intravenous epoprostenol in patients with functional class III and IV PAH.
Bosentan (Tracleer), an $\mathrm{ET}_{\mathrm{A}}$ and $\mathrm{ET}_{\mathrm{B}}$ receptor antagonist, is the first oral treatment approved for the treatment of PAH. Clinical studies showed that it improved haemodynamics and functional class, increased exercise capacity, and delayed clinical worsening compared with placebo in patients with primarily functional class III idiopathic PAH and PAH related to connective tissue diseases. ${ }^{56}$ Furthermore, the improvement in functional class with bosentan was often stable for at least 1 year. ${ }^{7}$ A recent prospective uncontrolled study of observed survival in patients with idiopathic PAH initially treated with bosentan, followed by other treatment if needed, also found improved survival compared with their predicted survival in the absence of targeted treatment. ${ }^{8}$ A possible long term effect of bosentan is suggested by experimental evidence that it can block and may reverse the pathological processes resulting from the excessive endothelin present in pulmonary and cardiac tissue. ${ }^{9}$ By blocking both $\mathrm{ET}_{\mathrm{A}}$ and $\mathrm{ET}_{\mathrm{B}}$ receptor subtypes, bosentan may alleviate abnormal vasoconstriction, prevent and reverse vascular hypertrophy and cardiac remodelling, attenuate pro-fibrotic and inflammatory

Abbreviations: mPAP, mean pulmonary arterial pressure; mRAP, mean right atrial pressure; $\mathrm{PAH}$, pulmonary arterial hypertension; PVR, pulmonary vascular resistance; PCWP, pulmonary capillary wedge pressure 
effects, and blunt neurohormonal activation. These effects may contribute to an improved survival in patients with PAH.

Because of its efficacy and oral availability, bosentan may be favoured as first line treatment before starting treatment with epoprostenol, especially in patients with less severe disease. However, it is unclear if a delay in starting epoprostenol treatment is detrimental to the long term outcome. This study was undertaken to determine whether initiating treatment with bosentan, followed by other treatment if needed, has any negative long term consequences compared with epoprostenol treatment. Recently available long term data from patients who received bosentan in clinical trials were compared with historical data from patients who were initially treated with epoprostenol at some of the same centres that participated in the bosentan studies.

\section{METHODS}

The study was conducted using two cohorts of patients with class III idiopathic PAH, one of which received bosentan as first line treatment in clinical trials (followed prospectively) and the other received epoprostenol as the initial treatment in the clinic (historical records). The analyses were confined to those patients with functional class III idiopathic PAH to reduce selection bias due to differences between the treatments.

\section{Bosentan cohort}

Patients included in the analyses were enrolled in two placebo controlled trials of bosentan in PAH. ${ }^{56}$ At entry, patients were $\geqslant 12$ years old and had severe symptomatic (World Health Organization (WHO) functional class III or IV) $\mathrm{PAH}$ with a resting mean pulmonary arterial pressure (mPAP) $>25 \mathrm{~mm} \mathrm{Hg}$, pulmonary vascular resistance (PVR) $>3 \mathrm{~mm} \mathrm{Hg} / \mathrm{l} / \mathrm{min}$, and pulmonary capillary wedge pressure (PCWP) $<15 \mathrm{~mm} \mathrm{Hg}$ as measured by right heart catheterisation, and 6 minute walk distance of 150-450 metres. Patients who completed a placebo controlled study were eligible to continue in an open label extension study. All patients gave written informed consent and the studies were conducted in accordance with the amended Declaration of Helsinki.

Of the 245 patients enrolled in the studies, 139 had class III idiopathic $\mathrm{PAH}$ and received bosentan either during a placebo controlled study or its extension. Patients had no previous exposure to epoprostenol or prostacyclin analogues, and the treating physician determined other treatments. During the placebo controlled studies bosentan was discontinued in patients starting on prostanoid treatment, but this was not required during the extensions. Data on mortality and alternative treatments were collected from September 1999 (start of the first bosentan study) to 31 December 2002 (data cut-off).

\section{Epoprostenol cohort}

Records of 785 patients started on epoprostenol from April 1987 to May 2002 were available from five referral centres (Clamart, France; Chicago, IL; Denver, CO; New York, NY; and San Diego, CA). The 346 patients with class III idiopathic $\mathrm{PAH}$ at the start of epoprostenol treatment, more than zero survival time, known survival status, and who started epoprostenol on or after January 1995 (considered contemporary) were included in the analyses. Data from only the first 36 months of treatment for each patient were included to parallel that available for bosentan treated patients. Where patient consent was not obtained, local Institutional Review Boards provided exempt approval for use of historical data, provided patient anonymity was maintained.

\section{Analysis of data}

Baseline and follow up information were summarised as mean (SD) or frequency counts and proportions for patients with available data. Baseline comparisons were performed using the Fisher's exact test (sex) and the Student's $t$ test (all others). Treatments at 12 and 24 months for bosentan treated patients were expressed as the proportions of patients followed for at least 12 and 24 months, respectively, omitting patients with an insufficient observation period.

Survival was assessed from the start of treatment to death or data cut-off if on bosentan, and to death, loss to follow up, or data cut-off if on epoprostenol. All patients with class III idiopathic PAH treated with bosentan were included in the analyses (intent to treat); patients lost to follow up were counted as dead at the last known contact. In contrast, patients on epoprostenol who were lost to follow up were censored at the last known contact. Observed survival data up to 36 months were reported as Kaplan-Meier estimates; the log rank test was used to explore the significance of the difference between treatments.

In order to explore possible underlying differences between the two treatment groups, two additional methods were used to compare the long term outcome of the different treatment regimens. The Cox proportional hazard regression model, ${ }^{10} \mathrm{a}$ statistical method used to model survival data in the presence of covariates and censoring, was used to adjust for differences between treatment groups at baseline. To confirm the results obtained with the Cox regression, a matched patient analysis was used. The objective of the analyses was to determine whether or not starting treatment with bosentan negatively influenced the long term outcome in patients with class III idiopathic PAH compared with starting with epoprostenol.

\section{Cox proportional hazard regression model}

The effect of treatment on survival was analysed using the Cox proportional hazard regression model adjusted for known prognostic factors such as relevant baseline factors identified empirically and/or in the literature. ${ }^{11}$ The stepwise variable selection procedure provided by the SAS procedure PHREG was used as a supportive approach and included age, sex, and baseline values of cardiac index, mPAP, mean right atrial pressure (mRAP), and PVR, with and without 6 minute walk distance as explanatory factors in the model. To address biases related to the dissimilar time periods of data collection in the two cohorts, the analyses were also performed on subsets of the epoprostenol cohort consisting of patients who started epoprostenol treatment on or after September 1999 (corresponding to bosentan treated patients) and between January 1995 and September 1999 (before bosentan was available as a treatment option). In all Cox regression analyses, hazard ratios represent the risk of death in the epoprostenol cohort versus the bosentan cohort and are presented with the associated $95 \%$ confidence limits and $\mathrm{p}$ value; a hazard ratio of $>1$ indicated that no negative influence of bosentan treatment was observed.

\section{Matched patient analysis}

The rules used to select two matched cohorts of class III patients are shown in the online supplement available on the Thorax website at www.thoraxjnl.com/supplemental. In brief, patients in each treatment group were matched using the baseline haemodynamic variables of cardiac index, mPAP, and mRAP. The procedure used strict matching criteria and conservative rules for eliminating unmatched patients. If within the match criteria there was an excess of bosentan patients, those with the best outcome were removed to equalise the number of patients in each treatment group. Conversely, if the excess was in the epoprostenol group, those 
Table 1 Demographic data and baseline characteristics of study patients

\begin{tabular}{|c|c|c|c|c|c|}
\hline & $\mathbf{n}$ & $\begin{array}{l}\text { Bosentan } \\
\text { cohort } \\
(N=139)\end{array}$ & $\mathbf{n}$ & $\begin{array}{l}\text { Epoprostenol } \\
\text { cohort } \\
(\mathrm{N}=346)\end{array}$ & p value* \\
\hline \multicolumn{5}{|l|}{ Age (years) } & 0.240 \\
\hline Mean (SD) & 139 & $46(16)$ & 341 & $41(14)$ & $<0.001$ \\
\hline Range & & $13-80$ & & $10-75$ & \\
\hline \multicolumn{6}{|c|}{$\begin{array}{l}\text { Time from diagnosis of PAH to start of } \\
\text { treatment (months)* }\end{array}$} \\
\hline Mean (SD) & 138 & $32(42)$ & 100 & $13(21)$ & $<0.001$ \\
\hline Range & & $0.3-326$ & & $0-135$ & \\
\hline \multicolumn{6}{|l|}{ Haemodynamics (mean (SD))† } \\
\hline Cardiac index $\left(1 / \mathrm{min} / \mathrm{m}^{2}\right)$ & 138 & $2.4(0.8)$ & 317 & $2.0(0.6)$ & $<0.001$ \\
\hline PVR (Wood units) & 132 & $12(6)$ & 207 & $18(10)$ & $<0.001$ \\
\hline $\mathrm{mPAP}(\mathrm{mm} \mathrm{Hg})$ & 139 & $56(15)$ & 333 & $66(18)$ & $<0.001$ \\
\hline mRAP $(\mathrm{mm} \mathrm{Hg})$ & 136 & $9(5)$ & 330 & $11(5)$ & $<0.001$ \\
\hline \multicolumn{6}{|l|}{ Walk test (m) } \\
\hline Mean (SD) & 139 & $351(80)$ & 171 & $335(106)$ & 0.136 \\
\hline \multicolumn{6}{|c|}{$\begin{array}{l}\text { mPAP, mean pulmonary artery pressure; mRAP, mean right atrial pressure; PAH, pulmonary arterial hypertension; } \\
\text { PVR, pulmonary vascular resistance; SD, standard deviation. } \\
\text { *p values were determined using the Fisher's exact test (sex) and the Student's } t \text { test (all others). } \\
\text { tFor most bosentan treated patients, time from diagnosis and haemodynamic data were available only at the start } \\
\text { of the placebo controlled study. }\end{array}$} \\
\hline
\end{tabular}

with the worst outcome were removed. This resulted in an equal number of patients in each cohort and closely matched baseline haemodynamics; any bias introduced by the procedure was against the bosentan cohort. Kaplan-Meier survival estimates and hazard ratios with 95\% confidence limits were computed for the resulting matched cohorts. As with the Cox regression analysis, a hazard ratio of $>1$ indicated no negative influence of bosentan treatment.

\section{RESULTS}

Several baseline factors differed between the 139 bosentan treated and 346 epoprostenol treated class III idiopathic PAH patients in the database (table 1). The epoprostenol cohort had more severe disease-that is, lower baseline cardiac index $\left(2.01 \vee 2.39 \mathrm{l} / \mathrm{min} / \mathrm{m}^{2}\right)$, higher PVR, mPAP, and mRAP, and a shorter time between diagnosis and initial treatment (13v32 months).

\section{Kaplan-Meier survival analysis}

The Kaplan-Meier estimates of survival after 1 and 2 years of treatment were $97 \%$ and $91 \%$, respectively, in the bosentan

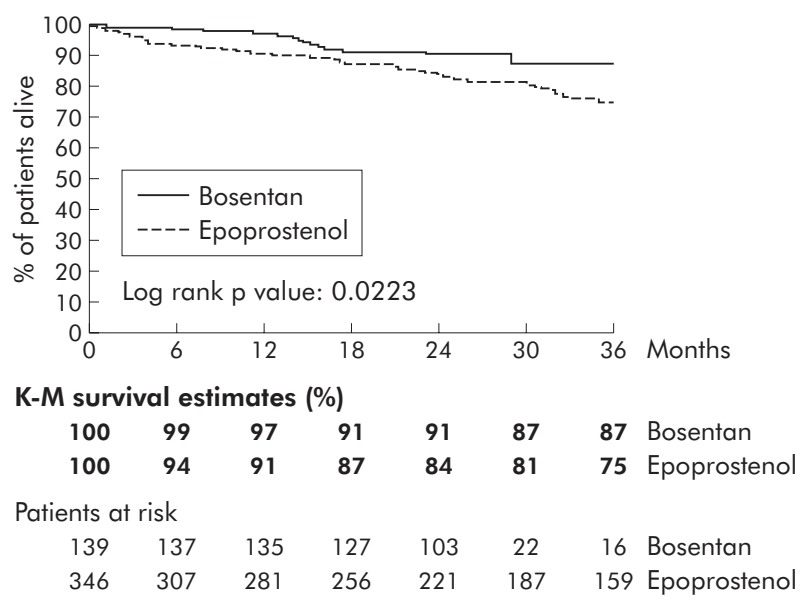

Figure 1 Kaplan-Meier (K-M) survival curves for patients with class III idiopathic PAH treated with bosentan (solid line) or epoprostenol (dashed line) cohort and $91 \%$ and $84 \%$ in the epoprostenol cohort (log rank $p=0.022$, fig 1$)$. Since the difference in survival may have been influenced by baseline differences, several methods were used to adjust the treatment effect for possible underlying differences between the two groups.

\section{Cox regression analysis}

The Cox regression was used to adjust for baseline factors that may cause the observed difference. Three different types of analyses using Cox regression were performed in the whole database (table 2) with the following results:

- When the model included no adjustment, the hazard ratio was $1.9(\mathrm{p}=0.025)$.

- When the model used the a priori defined set of clinically meaningful haemodynamic variables suggested by the literature, ${ }^{11}$ the treatment effect persisted (hazard ratio $2.2, p=0.014)$. Tests for variables such as the walk test or selected combinations (walk test + cardiac index, walk test + mRAP) produced similar results (hazard ratios 1.7-1.8, data not shown).

- When the stepwise variable selection was used, mPAP was selected by the model as a relevant explanatory variable but treatment effect again persisted with a hazard ratio of $2.3(\mathrm{p}=0.006)$. When the stepwise model was used on those patients with walk test results, both mPAP and walk test were selected as relevant explanatory variables and still the treatment effect persisted (hazard ratio 2.7, $\mathrm{p}=0.005)$

In all the Cox regression analyses performed, the probability of death was never higher in the bosentan cohort, regardless of the factors used for adjustment.

To address any bias resulting from the different time periods of data collection between the two cohorts-for example, different standards of care or a skewing of disease severity due to a new treatment option-the same analyses were performed on subgroups of epoprostenol treated patients (table 2). The bosentan cohort was compared with patients who started epoprostenol on or after September 1999 (as in the bosentan cohort) and with those who started epoprostenol between January 1995 and September 1999 (before bosentan was a treatment option). Results from all analyses using both subsets of the epoprostenol cohort support the results of the main analyses-that is, the hazard 
Table 2 Main Cox regression analyses on mortality for class III idiopathic PAH patients treated with bosentan or epoprostenol

\begin{tabular}{|c|c|c|c|c|c|c|}
\hline & $\begin{array}{l}\text { Bosentan } \\
\text { (n) }\end{array}$ & $\begin{array}{l}\text { Epoprostenol } \\
\text { (n) }\end{array}$ & No of events & Hazard ratio & $95 \% \mathrm{CL}$ & p value \\
\hline \multicolumn{7}{|l|}{ Entire database* } \\
\hline No adjustment & 139 & 346 & 85 & 1.9 & $1.1,3.5$ & 0.025 \\
\hline Model adjusted for haemodynamics§ & 136 & 314 & 76 & 2.2 & $1.2,4.0$ & 0.014 \\
\hline $\begin{array}{l}\text { Stepwise model } \dagger \\
\text { mPAP }\end{array}$ & 136 & 314 & 76 & 2.3 & $1.3,4.2$ & 0.006 \\
\hline $\begin{array}{l}\text { Stepwise model } \ddagger \\
\text { mPAP, walk test }\end{array}$ & 136 & 169 & 46 & 2.7 & $1.4,5.4$ & 0.005 \\
\hline \multicolumn{7}{|l|}{ Patients treated during same time period ${ }^{*}$} \\
\hline No adjustment & 139 & 81 & 26 & 2.5 & $1.1,5.6$ & 0.022 \\
\hline $\begin{array}{l}\text { Model adjusted for haemodynamics§ } \\
\text { Stepwise modelt }\end{array}$ & 136 & 78 & 25 & 2.3 & $0.9,5.6$ & 0.073 \\
\hline $\begin{array}{l}\text { Stepwise modelt } \\
\text { None }\end{array}$ & 136 & 78 & 25 & 2.4 & $1.1,5.4$ & 0.033 \\
\hline $\begin{array}{l}\text { Stepwise model } \\
\text { Walk test }\end{array}$ & 136 & 29 & 18 & 2.1 & $0.7,6.6$ & 0.206 \\
\hline \multicolumn{7}{|l|}{ Patients treated during different time periods* } \\
\hline No adjustment & 139 & 217 & 67 & 1.9 & $1.0,3.5$ & 0.035 \\
\hline Model adjusted for haemodynamics§ & 136 & 196 & 60 & 2.2 & $1.2,4.3$ & 0.016 \\
\hline $\begin{array}{l}\text { Stepwise model } \dagger \\
\text { mPAP, mRAP }\end{array}$ & 136 & 196 & 60 & 2.3 & $1.2,4.3$ & 0.012 \\
\hline $\begin{array}{l}\text { Stepwise model } \neq \\
\text { mPAP, mRAP }\end{array}$ & 136 & 117 & 39 & 2.7 & $1.3,5.7$ & 0.007 \\
\hline
\end{tabular}

$\mathrm{CL}$, confidence limits; mPAP, mean pulmonary artery pressure; mRAP, mean right atrial pressure.

*In the entire database, patients started bosentan on or after September 1999 and epoprostenol on or after January 1995. For the same time period all patients started treatment on or after September 1999, and for different time periods patients started bosentan on or after September 1999 and started epoprostenol between January 1995 and September 1999.

†Using the parameters age, sex, and baseline cardiac index, mPAP, mRAP, pulmonary vascular resistance, and WHO functional class.

\#Using the stepwise model parameters given in the footnote above + walk test.

§arameters of cardiac index, mPAP, and mRAP suggested by the literature."

ratios for mortality ranged from 1.6 to 2.7 (including analyses not shown), with the greater risk consistently in the epoprostenol treated patients.

\section{Matched patient analysis}

A more empirical method was used to reduce any underlying differences between the two groups. This required the identification of matched cohorts of patients selected on the basis of their haemodynamic variables and using exclusion rules consistently biased against bosentan. The selected analysis set contained two cohorts of 83 patients
( $60 \%$ of bosentan patients and $24 \%$ of epoprostenol patients) that were well matched for demographic data and baseline haemodynamic and exercise variables (table 3). KaplanMeier survival estimates in the two matched cohorts were nearly identical (fig 2, hazard ratio 1.03).

\section{Overall outcomes}

During the entire data collection period overall outcomes in the two cohorts of class III idiopathic PAH patients were different. The mean (SD) duration of observation in the epoprostenol cohort was 3.1 (2.4) years, during which time

Table 3 Demographic data and baseline characteristics of class III idiopathic PAH patients in matched cohorts

\begin{tabular}{|c|c|c|c|c|c|}
\hline & $N$ & $\begin{array}{l}\text { Bosentan matched } \\
\text { cohort } \\
(\mathrm{N}=83)\end{array}$ & $\mathbf{n}$ & $\begin{array}{l}\text { Epoprostenol } \\
\text { matched cohort } \\
(\mathrm{N}=83)\end{array}$ & p value* \\
\hline $\operatorname{Sex}(M / F, \%)$ & 83 & $24 / 76$ & 83 & $28 / 72$ & 0.723 \\
\hline \multicolumn{6}{|l|}{ Age (years) } \\
\hline Mean (SD) & 83 & $46(16)$ & 83 & $43(13)$ & 0.136 \\
\hline Range & & $13-76$ & & $15-74$ & \\
\hline \multicolumn{6}{|l|}{ Time from diagnosis (months)* } \\
\hline $\begin{array}{l}\text { Mean (SD) } \\
\text { Range }\end{array}$ & 83 & $29(36)$ & 26 & $11(19)$ & 0.014 \\
\hline \multicolumn{6}{|l|}{ Haemodynamics (mean (SD)) $†$} \\
\hline Cardiac index $\left(1 / \mathrm{min} / \mathrm{m}^{2}\right)$ & 83 & $2.2(0.6)$ & 83 & $2.1(0.6)$ & 0.610 \\
\hline PVR (Wood units) & 79 & $13(6)$ & 53 & $14(6)$ & 0.553 \\
\hline mPAP $(\mathrm{mm} \mathrm{Hg})$ & 83 & $57(15)$ & 83 & $59(15)$ & 0.397 \\
\hline mRAP (mm Hg) & 83 & $10(5)$ & 83 & $10(5)$ & 0.893 \\
\hline \multicolumn{6}{|l|}{ Walk test $(\mathrm{m})$} \\
\hline Mean (SD) & 83 & $355(76)$ & 41 & $350(110)$ & 0.762 \\
\hline
\end{tabular}

mPAP, mean pulmonary artery pressure; mRAP, mean right atrial pressure; $\mathrm{PAH}$, pulmonary arterial hypertension; PVR, pulmonary vascular resistance; $S D$, standard deviation.

Patients were matched using baseline cardiac index, mPAP, and mRAP (see Methods section in online supplement). * $p$ values were determined using the Fisher's exact test (sex) and the Student's $t$ test (all others).

tFor most bosentan treated patients, time from diagnosis and haemodynamic data were available only at the start of the placebo controlled study. 


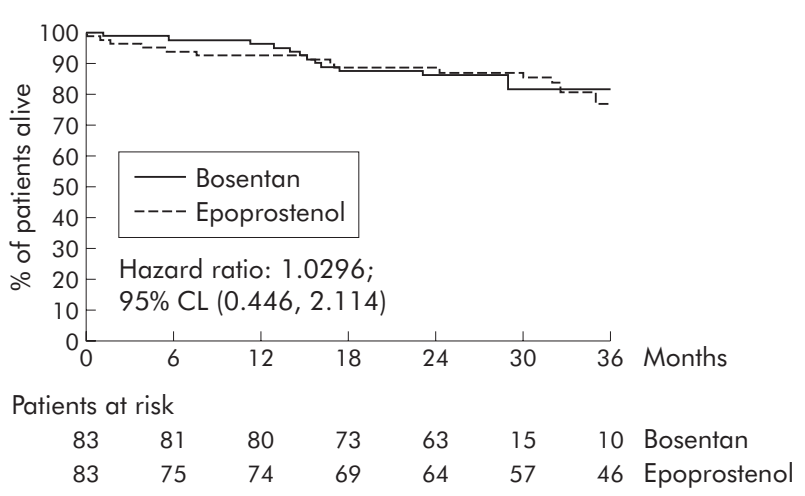

Figure 2 Kaplan-Meier survival curves for matched cohorts of class III idiopathic PAH patients treated with bosentan (solid line) or epoprostenol (dashed line). CL, confidence limits.

71 of the 346 patients died and 22 underwent lung transplantation. The bosentan cohort was followed for a shorter mean (SD) time of $2.2(0.5)$ years and had fewer deaths (13/139) and lung transplantations (2/139) than the epoprostenol cohort. Furthermore, patients in the bosentan cohort had the option of being transferred to or given other drug treatments in addition at the discretion of the investigator. However, after 1 year of follow up, $87 \%$ of the bosentan treated patients remained on bosentan alone (table 4). Epoprostenol was added to bosentan in five patients and replaced it in two others. After 2 years, $75 \%$ of the 116 patients followed for a full 2 years were still being treated with bosentan alone. By this time a further two patients had been transferred to epoprostenol. Other treatment changes were infrequent but included the addition of intravenous or inhaled iloprost, subcutaneous treprostinil, or oral sildenafil; replacement therapy with subcutaneous treprostinil or oral sitaxsentan; and discontinuation of bosentan without replacement.

\section{DISCUSSION}

Long term treatment with epoprostenol is known to improve symptoms, quality of life, exercise capacity, haemodynamics, and survival in patients with class III or IV idiopathic PAH. ${ }^{137}$ Despite its efficacy, epoprostenol remains a complicated, inconvenient and costly treatment. In addition, it has several drawbacks including complications related to its delivery system (indwelling venous catheter related infections, portable pump dysfunction) as well as side effects including headache, jaw pain, diarrhoea, and rash. In contrast, the side effect profile of oral bosentan includes an increased incidence of oedema and raised liver enzymes. ${ }^{67}$ Bosentan has been approved in North America and Europe for the treatment of PAH (WHO functional class III-IV), and for many clinicians bosentan provides an appealing alternative to epoprostenol as first line treatment. However, clinicians are faced with the difficult decision of choosing between an agent that may be more convenient for the patient (bosentan) and one that has demonstrated improved survival (epoprostenol).

The best way to compare the long term effects of different therapeutic approaches is a prospective, randomised, controlled study comparing the two interventions. However, conducting a parallel arm, randomised trial of intravenous epoprostenol versus oral bosentan would be difficult because of the inability to blind the study, bias introduced by patient preferences, and the poorly defined target population at the present time (epoprostenol is most often administered to patients with more severe disease while bosentan may be
Table 4 Mortality and treatment at 12 and 24 months of follow up in patients in the bosentan cohort

\begin{tabular}{|c|c|c|}
\hline & $\begin{array}{l}12 \text { months } \\
(\mathrm{N}=139)\end{array}$ & $\begin{array}{l}24 \text { months } \\
(\mathrm{N}=139)\end{array}$ \\
\hline Dead & $4(2.9 \%)^{*}$ & $13(9.4 \%)^{*}$ \\
\hline Alive & $135(97.1 \%)$ & $103(74.1 \%)$ \\
\hline Insufficient observation & - & $23(16.5 \%)$ \\
\hline Patients with sufficient & $(n=139)$ & $(n=116)$ \\
\hline observation timeł & & \\
\hline Bosentan alone & $121(87 \%)$ & $87(75 \%)$ \\
\hline Bosentan + other treatment & $7(5 \%)$ & $8(7 \%)$ \\
\hline Other or no treatment & $7(5 \%) \dagger$ & $8(7 \%) \dagger$ \\
\hline Dead & $4(3 \%)^{*}$ & $13(11 \%)^{*}$ \\
\hline \multicolumn{3}{|c|}{$\begin{array}{l}\text { *Includes one patient lost to follow up. } \\
\text { tIncludes patients with missing information. } \\
\text { \$Percentages are based on the total number of patients with sufficient } \\
\text { observation time at the given time point. }\end{array}$} \\
\hline
\end{tabular}

started in patients who are less ill). A comparison of existing survival data from two cohorts of patients, one initially treated with bosentan and one initially started on epoprostenol, was therefore considered an appropriate approach to address the question.

Because of the retrospective nature of the study, several analyses were performed to adjust for any inherent differences between the cohorts that may bias the results. The Cox regression was used to control for explanatory variables other than treatment effect. As with other regression models (such as multiple linear regression), the Cox proportional hazard regression model provides an adjustment of the treatment effect based on known prognostic factors, thus allowing a correct interpretation of the results even in the presence of an imbalance of the prognostic factors. Baseline haemodynamic data suggested that the epoprostenol cohort had more severe disease. The results of the regression analysis that adjusted for this difference were consistent; the probability of death was never greater in the bosentan cohort than in the epoprostenol cohort. Since epoprostenol was available for therapeutic use before bosentan, the standard of care might have been different in the two cohorts. This bias was addressed by comparing patients treated during the same time period (from September 1999 to data cut-off). Also, the availability of bosentan as an investigational drug after September 1999 might have influenced treatment decisions, with epoprostenol proposed for only the most severe patients. This was addressed by comparing the bosentan cohort with patients treated with epoprostenol before September 1999. These analyses showed similar results in that the probability of death was never greater in the bosentan cohort than in the epoprostenol cohort. To address differences in patient selection in the two cohorts (clinical studies $v$ the clinic), matched cohorts were selected using rules intentionally biased against the bosentan cohort. Survival estimates of these matched cohorts were nearly identical with a hazard ratio of 1.03 .

In summary, Kaplan-Meier estimates of survival in the bosentan cohort were not inferior to those of the epoprostenol cohort. Cox regression analyses to adjust for prognostic factors and time periods confirmed this result, regardless of the factor or time period considered. Kaplan-Meier estimates of survival for the conservatively biased matched cohorts were very similar (hazard ratio 1.03). Since this was a retrospective study, the significance of the findings must be interpreted with caution. The different statistical approaches were successively more conservative and even biased against bosentan. They resulted in a reduction in the difference initially seen between the two cohorts. However, in no case was the probability of survival in the bosentan cohort less 
than in the epoprostenol cohort. Since epoprostenol was a treatment option in the bosentan cohort, the results of this study might have been biased by the early use of epoprostenol in bosentan patients. However, 2 years after treatment initiation $75 \%$ of the bosentan cohort remained on bosentan monotherapy. In addition, the longer time from diagnosis to initial treatment in the bosentan cohort may indicate more stable disease and slower progression compared with the epoprostenol cohort.

There are a number of limitations to this type of analysis. Data from the two treatment cohorts were obtained under different conditions (protocol guided studies in the case of bosentan and needs based treatment in the clinic in the case of epoprostenol). This difference not only affects the type of treatment administered but also the type of patient included in each cohort. Treatment with epoprostenol was based on practices at each centre, which vary centre to centre, and data were not systematically collected at these centres in the manner specified for the clinical trials. Statistical methods were used to address many of the possible resulting biases, but the 6 minute walk test was not included in the identification of matched cohorts since the assessment was available for only $42 \%$ of patients treated with epoprostenol. Fewer patients in the bosentan cohort than in the epoprostenol cohort were followed for 3 years and, arguably, the long term effect of treatment would be more in evidence with even longer follow up. With long term data collection, some of the data from both the epoprostenol and bosentan cohorts included in this study have been previously reported in other ${\text { analyses. }{ }^{1} 27}^{7}$

The use of bosentan as first line treatment in patients with PAH could delay the initiation of epoprostenol, a treatment that has been shown to confer a survival benefit in this disease. No evidence was found in the present study to suggest that initial treatment with oral bosentan-followed by or with the addition of other treatment if clinically indicated-adversely affected the long term outcome compared with initial intravenous epoprostenol in patients with class III idiopathic PAH. Decisions regarding treatment initiation for individual idiopathic patients should take into account a number of factors, including ease of administration, side effect profile, and long term outcomes associated with each treatment option. Ideally, prospective comparison studies are warranted in order to provide treating physicians with additional information.

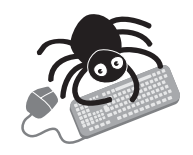

Details of the selection of cohorts for matched patient analysis are shown in the online supplement available on the Thorax website at http://www.thoraxjnl.com/ supplemental
Authors' affiliations

O Sitbon, M Humbert, G Simonneau, Antoine Béclère Hospital, Paris-

Sud University, Clamart, France

V V McLaughlin, University of Michigan, Ann Arbor, MI, USA

D B Badesch, University of Colorado Health Sciences Center, Denver, CO, USA

R J Barst, Columbia University College of Physicians and Surgeons, New York, NY, USA

C Black, Royal College of Physicians, London, UK

N Galiè, University of Bologna, Bologna, Italy

M Rainisio, Actelion Pharmaceuticals Ltd, Allschwil, Switzerland

L J Rubin, University of California, San Diego, CA, USA

This study was supported by a grant from Actelion Pharmaceuticals Ltd, Allschwil, Switzerland.

Competing interests: OS, WVM, DBB, RJB, CB, NG, MH, LR, and GS have been remunerated consultants for Actelion Pharmaceuticals Ltd, the manufacturer of bosentan; MR is an employee of Actelion Pharmaceuticals Ltd.

Contributing investigators: A Keogh (Australia); M Delcroix (Belgium); M Humbert, G Simonneau, O Sitbon (France); F Kleber (Germany); I BenDov (Israel); N Galiè (Italy); T Pulido (Mexico); A Torbicki (Poland); M Gomez-Sanchez (Spain); C Black, AJ Peacock, J Pepke-Zaba (UK); D Badesch, R Barst, R Channick, B DeBoisblanc, T DeMarco, R Doyle, E Horn, R Frantz, A Frost, M Landzberg, V McLaughlin, S Murali, R Oudiz, I Robbins, U Rubin, R Schilz, V Tapson (USA).

\section{REFERENCES}

1 McLaughlin VV, Shillington A, Rich S. Survival in primary pulmonary hypertension: the impact of epoprostenol therapy. Circulation 2002; 106: 1477-82.

2 Sitbon $\mathrm{O}$, Humbert $M$, Nunes $\mathrm{H}$, et al. Long-term intravenous epoprostenol infusion in primary pulmonary hypertension: prognostic factors and survival. J Am Coll Cardiol 2002;40:780-8.

3 Barst RJ, Rubin $\sqcup$, Long WA, et al. A comparison of continuous intravenous epoprostenol (prostacyclin) with conventional therapy for primary pulmonary hypertension. N Engl J Med 1996;334:296-301.

4 Badesch DB, Tapson VF, McGoon MD, et al. Continuous intravenous epoprostenol for pulmonary hypertension due to the scleroderma spectrum of disease. A randomized, controlled trial. Ann Intern Med 2000;132:425-34.

5 Channick RN, Simonneau G, Sitbon O, et al. Effects of the dual endothelinreceptor antagonist bosentan in patients with pulmonary hypertension: a randomised placebo-controlled study. Lancet 2001;358:1119-23.

6 Rubin LJ, Badesch DB, Barst RJ, et al. Bosentan therapy for pulmonary arterial hypertension. N Engl J Med 2002;346:896-903.

7 Sitbon O, Badesch DB, Channick RN, et al. Effects of the dual endothelin receptor antagonist bosentan in patients with pulmonary arterial hypertension. A 1-year follow-up study. Chest 2003;124:247-54.

8 Mclaughlin VV, Sitbon O, Rubin $\amalg$, et al. Survival with first-line bosentan in patients with primary pulmonary hypertension. Eur Respir J 2005;25:244-9.

9 Clozel M. Effects of bosentan on cellular processes involved in pulmonary arterial hypertension: do they explain the long-term benefit? Ann Med 2003;35: 1-9.

10 Cox DR, Oakes D. Analysis of survival data. London: Chapman and Hall, 1984.

11 D'Alonzo GE, Barst RJ, Ayres SM, et al. Survival in patients with primary pulmonary hypertension. Results from a national prospective registry. Ann Intern Med 1991;115:343-9. 